Supplement of Atmos. Chem. Phys., 17, 9223-9236, 2017

https://doi.org/10.5194/acp-17-9223-2017-supplement

(C) Author(s) 2017. This work is distributed under

the Creative Commons Attribution 3.0 License.

(c) (1)

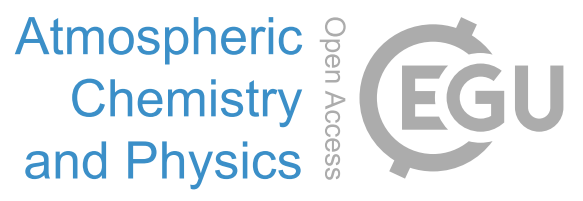

Supplement of

\title{
Wildfire air pollution hazard during the 21st century
}

\section{Wolfgang Knorr et al.}

Correspondence to: Wolfgang Knorr (wolfgang.knorr@nateko.lu.se)

The copyright of individual parts of the supplement might differ from the CC BY 3.0 License. 


\section{$1 \quad$ Supplementary Information}

2 Table S1: Countries/regions used for scaling GFED4.1s wildfire emissions.

\begin{tabular}{|c|c|c|c|c|}
\hline Code & Country name & $\begin{array}{l}\text { World } \\
\text { region }\end{array}$ & $\begin{array}{l}\text { grid cells on } \\
\text { 1-degree } \\
\text { grid }\end{array}$ & $\begin{array}{c}\text { non-crop } \\
\text { cells }^{1}\end{array}$ \\
\hline$\overline{A G O}$ & Angola & & 100 & 100 \\
\hline BEN & Benin & & 10 & 6 \\
\hline BWA & Botswana & & 46 & 46 \\
\hline BFA & Burkina Faso & & 23 & 21 \\
\hline BDI & Burundi & & 3 & 3 \\
\hline CMR & Cameroon & & 37 & 35 \\
\hline CAF & Central African & & 49 & 46 \\
\hline CAF & Republic & & 100 & 100 \\
\hline $\begin{array}{l}\text { TCD } \\
\text { COG }\end{array}$ & Chad & & 24 & 24 \\
\hline & Congo, Dem. & & & \\
\hline$\angle A R$ & Republic & & 176 & 176 \\
\hline CIV & Cote d'Ivoire & & 25 & 24 \\
\hline ERI & Eritrea & & 12 & 12 \\
\hline ETH & Ethiopia & & 90 & 89 \\
\hline GAB & Gabon & & 20 & 20 \\
\hline GHA & Ghana & & 18 & 13 \\
\hline GIN & Guinea & & 20 & 20 \\
\hline GNB & Guinea-Bissau & & 1 & 1 \\
\hline KEN & Kenya & Sub- & 41 & 40 \\
\hline LSO & Lesotho & Saharan & 1 & 1 \\
\hline LBR & Liberia & & 5 & 5 \\
\hline MDG & Madagascar & & 43 & 43 \\
\hline MWI & Malawi & & 10 & 10 \\
\hline MLI & Mali & & 106 & 106 \\
\hline MRT & Mauritania & & 80 & 80 \\
\hline MOZ & Mozambique & & 61 & 60 \\
\hline NAM & Namibia & & 66 & 66 \\
\hline NER & Niger & & 94 & 80 \\
\hline NGA & Nigeria & & 74 & 40 \\
\hline SEN & Senegal & & 17 & 16 \\
\hline SLE & Sierra Leone & & 5 & 5 \\
\hline SOM & Somalia & & 55 & 55 \\
\hline ZAF & South Africa & & 99 & 98 \\
\hline SDN & Sudan & & 207 & 199 \\
\hline TGO & Togo & & 2 & 1 \\
\hline UGA & Uganda & & 16 & 13 \\
\hline TZA & Tanzania & & 73 & 72 \\
\hline ZMB & Zambia & & 63 & 63 \\
\hline ZWE & Zimbabwe & & 30 & 30 \\
\hline DZA & Algeria & & 189 & 184 \\
\hline EGY & Egypt & & 77 & 76 \\
\hline LBY & Libya & & 131 & 131 \\
\hline MAR & Morocco & & 56 & 49 \\
\hline TUN & Tunisia & Developing & 14 & 11 \\
\hline AFG & Afghanistan & Middle East & 53 & 52 \\
\hline IRN & Iran & and North & 134 & 129 \\
\hline IRQ & Iraq & Africa & 37 & 31 \\
\hline JOR & Jordan & (DMNA) & 6 & 6 \\
\hline SAU & Saudi Arabia & & 154 & 154 \\
\hline SYR & Syria & & 15 & 9 \\
\hline TUR & Turkey & & 57 & 44 \\
\hline YEM & Yemen & & 31 & 31 \\
\hline$\overline{\text { AUT }}$ & Austria & & 7 & 7 \\
\hline BNL & Benelux & & 5 & 3 \\
\hline CRS & $\begin{array}{l}\text { Croatia and } \\
\text { Slovenia }\end{array}$ & & 3 & 2 \\
\hline CZE & Czech Republic & & 5 & 3 \\
\hline DNK & Denmark & & 6 & 3 \\
\hline EST & Estonia & & 4 & 4 \\
\hline FIN & Finland & & 28 & 27 \\
\hline FRA & France & & 41 & 24 \\
\hline DEU & Germany & & 32 & 29 \\
\hline GRC & Greece & High- & 10 & 9 \\
\hline HUN & Hungary & income & 7 & 2 \\
\hline ISL & Iceland & Europe & 7 & 7 \\
\hline IRL & Ireland & (HEUR) & 5 & 4 \\
\hline ITA & Italy & & 23 & 11 \\
\hline NOR & Norway & & 31 & 31 \\
\hline $\mathrm{POL}$ & Poland & & 25 & 11 \\
\hline PRT & Portugal & & 6 & 5 \\
\hline SVK & Slovakia & & 6 & 2 \\
\hline ESP & Spain & & 40 & 24 \\
\hline SWE & Sweden & & 39 & 39 \\
\hline CHE & Switzerland & & 2 & 2 \\
\hline GBR & $\begin{array}{l}\text { United } \\
\text { Kingdom }\end{array}$ & & 19 & 13 \\
\hline
\end{tabular}

${ }^{1}$ Cells with less than $50 \%$ cropland fraction in past or future scenarios

${ }^{2}$ Constant emissions assumed because dominated by croplands

${ }^{3}$ Constant emissions assumed because zero current wildfire emissions in some simulations

\begin{tabular}{|c|c|c|c|c|c|c|}
\hline Code & Country name & $\begin{array}{l}\text { World } \\
\text { region }\end{array}$ & $\begin{array}{c}\text { grid cells } \\
\text { on 1- } \\
\text { degree grid }\end{array}$ & $\begin{array}{c}\text { non-crop } \\
\text { cells }\end{array}$ & $\begin{array}{c}\text { longitude } \\
\text { range }\end{array}$ & $\begin{array}{c}\text { latitude } \\
\text { range }\end{array}$ \\
\hline ARM & Armenia & & 4 & 4 & & \\
\hline AZE & Azerbaijan & & 7 & 6 & & \\
\hline GEO & Georgia & & 6 & 6 & & \\
\hline KAZ & Kazakstan & & 213 & 206 & & \\
\hline KGZ & Kyrgyzstan & & 16 & 16 & & \\
\hline TJK & Tajikistan & & 8 & 8 & & \\
\hline TKM & Turkmenistan & & 35 & 35 & & \\
\hline UZB & Uzbekistan & & 35 & 32 & & \\
\hline BLR & Belarus & Eastern & 16 & 14 & & \\
\hline BGR & Bulgaria & $\begin{array}{l}\text { Europe, } \\
\text { Euscia and }\end{array}$ & 10 & 8 & & \\
\hline LLI & Latvia and Lithunania & $\begin{array}{l}\text { Russia and } \\
\text { Rentra }\end{array}$ & 10 & 5 & & \\
\hline ROM & Romania & $\begin{array}{l}\text { Central Asia } \\
\text { (ERC) }\end{array}$ & 19 & 10 & & \\
\hline RUS-SW & & & 78 & 32 & $W$ of $60^{\circ} \mathrm{E}$ & $\mathrm{S}$ of $52^{\circ} \mathrm{N}$ \\
\hline RUS-NW & & & 212 & 150 & $W$ of $55^{\circ} \mathrm{E}$ & $\mathrm{N}$ of $52^{\circ} \mathrm{N}$ \\
\hline RUS-C & Russian Federation & & 553 & 506 & not in ot & ther RUS \\
\hline RUS-SE & & & 232 & 232 & $\mathrm{E}$ of $110^{\circ} \mathrm{E}$ & $\mathrm{S}$ of $60^{\circ} \mathrm{N}$ \\
\hline RUS-NE & & & 327 & 327 & $\mathrm{E}$ of $110^{\circ} \mathrm{E}$ & $\mathrm{N}$ of $60^{\circ} \mathrm{N}$ \\
\hline UKR & Ukraine & & 47 & 5 & & \\
\hline YUA & $\begin{array}{l}\text { Serbia, Montenegro, } \\
\text { Bosnia, Macedonia }\end{array}$ & & 13 & 11 & & \\
\hline $\mathrm{CHN}-\mathrm{W}$ & & & 348 & 342 & W of $105^{\circ} \mathrm{E}$ & \\
\hline CHN-E & China & Developing & 283 & 211 & $\mathrm{E}$ of $105^{\circ} \mathrm{E}$ & $\mathrm{S}$ of $43^{\circ} \mathrm{N}$ \\
\hline CHN-N & & East Asia & 122 & 103 & & $\mathrm{~N}$ of $43^{\circ} \mathrm{N}$ \\
\hline PRK & North Korea & (DEAS) & 10 & 8 & & \\
\hline MNG & Mongolia & & 131 & 131 & & \\
\hline BTN & Bhutan & & 4 & 2 & & \\
\hline KHM & Cambodia & & 14 & 13 & & \\
\hline IND & India & & 256 & 50 & & \\
\hline IDN & Indonesia & & 125 & 116 & & \\
\hline LAO & Laos & & 18 & 18 & & \\
\hline MYS & Malaysia & & 23 & 23 & & \\
\hline MMR & Myanmar & South and & 44 & 36 & & \\
\hline NPL & Nepal & Asia (SSEA) & 12 & 8 & & \\
\hline PAK & Pakistan & Asia (SSEA) & 58 & 44 & & \\
\hline PHL & Philippines & & 16 & 14 & & \\
\hline LKA & Sri Lanka & & 4 & 4 & & \\
\hline THA & Thailand & & 42 & 30 & & \\
\hline VNM & Viet Nam & & 27 & 22 & & \\
\hline PNG & Papua New Guinea & & 31 & 31 & & \\
\hline AUS-SW & & & 18 & 16 & $W$ of $120^{\circ} \mathrm{E}$ & $S$ of $30^{\circ} \mathrm{S}$ \\
\hline AUS-E & Australia & $\begin{array}{l}\text { Australla } \\
\text { and New }\end{array}$ & 200 & 178 & $\mathrm{E}$ of $140^{\circ} \mathrm{E}$ & $\mathrm{S}$ of $18^{\circ} \mathrm{S}$ \\
\hline AUS-C & Australla & Zealand & 317 & 316 & not in ot & ther AUS \\
\hline AUS-N & & Zealana & 76 & 76 & & $\mathrm{~N}$ of $18^{\circ} \mathrm{S}$ \\
\hline NZL & New Zealand & & 22 & 22 & & \\
\hline CAN-W & & & 385 & $341 \mathrm{~V}$ & $W$ of $100^{\circ} \mathrm{W}$ & \\
\hline CAN-C & Canada & & 192 & 185 & $100 \ldots 80^{\circ} \mathrm{W}$ & \\
\hline CAN-E & & America & 176 & 176 & $\mathrm{E}$ of $80^{\circ} \mathrm{W}$ & \\
\hline USA-W & & America & 314 & 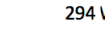 & $\mathrm{W}$ of $100^{\circ} \mathrm{W}$ & \\
\hline USA-E & America & & 372 & 222 & $\mathrm{E}$ of $100^{\circ} \mathrm{W}$ & \\
\hline ALK & & & 116 & 116 & & $\mathrm{~N}$ of $50^{\circ} \mathrm{N}$ \\
\hline 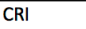 & Costa Rica & & 3 & 3 & & \\
\hline CUB & Cuba & & 7 & 5 & & \\
\hline DOM & Dominican Republic & & 4 & 4 & & \\
\hline GTM & Guatemala & & 15 & 15 & & \\
\hline $\mathrm{HTI}$ & Haiti & & 2 & 2 & & \\
\hline HND & Honduras & & 9 & 9 & & \\
\hline MEX-W & Mexica & & 120 & 115 & $\mathrm{~W}$ of $95^{\circ} \mathrm{W}$ & \\
\hline MEX-SE & Wexico & & 19 & 19 & $\mathrm{E}$ of $95^{\circ} \mathrm{W}$ & \\
\hline NIC & Nicaragua & & 8 & 8 & & \\
\hline PAN & Panama & & 6 & 6 & & \\
\hline ARG & Argentina & & 230 & 207 & & \\
\hline $\mathrm{BOL}$ & Bolivia & & 88 & 88 & & \\
\hline BRA-W & & America and & 197 & 192 & $W$ of $49^{\circ} \mathrm{W}$ & $S$ of $5^{\circ} \mathrm{S}$ \\
\hline BRA-E & Brazil & Cariobean & 316 & 294 & $\mathrm{E}$ of $49^{\circ} \mathrm{W}$ & \\
\hline BRA-N & & & 161 & 161 & & $\mathrm{~N}$ of $5^{\circ} \mathrm{S}$ \\
\hline CHL & Chile & & 61 & 60 & & \\
\hline $\mathrm{COL}$ & Colombia & & 88 & 88 & & \\
\hline ECU & Ecuador & & 19 & 19 & & \\
\hline GUF & French Guiana & & 6 & 6 & & \\
\hline GUY & Guyana & & 15 & 15 & & \\
\hline PRY & Paraguay & & 28 & 28 & & \\
\hline PER & Peru & & 100 & 100 & & \\
\hline SUR & Suriname & & 11 & 11 & & \\
\hline URY & Uruguay & & 15 & 15 & & \\
\hline VEN & Venezuela & & 73 & 73 & & \\
\hline ISR & Israel & & 4 & 4 & & \\
\hline JPN & Japan & & 28 & 28 & & \\
\hline KOR & South Korea & & 6 & 6 & & \\
\hline OMN & Oman & & 26 & 26 & & \\
\hline ARE & United Arab Emirates & - & 8 & 8 & & \\
\hline MDA & Republic of Moldova ${ }^{2}$ & & 4 & 0 & & \\
\hline BGD & Bangladesh $^{2}$ & & 10 & 1 & & \\
\hline GRL & Greenland $^{3}$ & & 31 & 31 & & \\
\hline
\end{tabular}


$4 \quad$ Table S2: Current PM 2.5 Emissions [Gg/yr] by world region from various sources

$\begin{array}{lrrrr}\text { Region } & \text { Wildfire } & \text { Deforestation } & \text { Peat Fire } & \text { Anthropogenic } \\ \text { SSA } & 14,973 & 538 & 0 & 5,864 \\ \text { LAC } & 3,138 & 1,886 & 0 & 2,534 \\ \text { ERCA } & 2,832 & 0 & 18 & 2,490 \\ \text { SSEA } & 1,593 & 1,499 & 598 & 10,392 \\ \text { AUN } & 1,536 & 22 & 0 & 186 \\ \text { NOA } & 1,349 & 0 & 30 & 1,462 \\ \text { DEAS } & 364 & 17 & 0 & 13,324 \\ \text { HEUR } & 31 & 0 & 0 & 1,630 \\ \text { DMNA } & 7 & 0 & 0 & 1,561 \\ \text { Global } & 25,842 & 3,968 & 646 & 40,370\end{array}$

5 SSA: Sub-Saharan Africa, LAC: Latin America \& Caribbean, ERCA: Eastern Europe, Russia \& Central Asia, SSEA: South \& Southeast Asia, AUN: Australia \& New Zealand, NOA: North America, DEAS: Developing East Asia, HEUR: High-income Europe, DMNA: Developing Middle-East \& North Africa. 


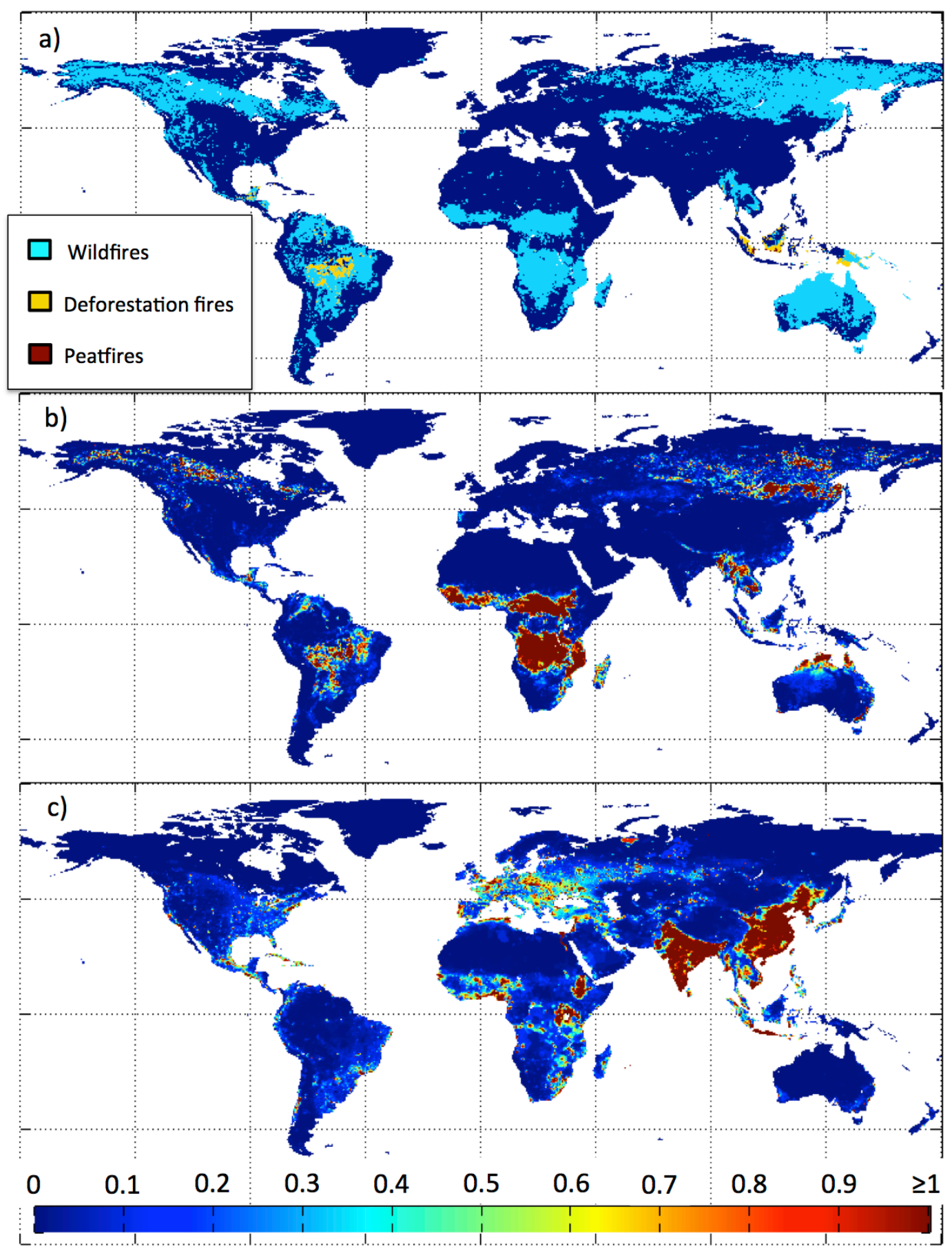

Figure S1: a) Largest current source of PM 2.5 emissions (dark blue areas: either emissions are zero, or anthropogenic emissions are the largest source); b) current wildfire and c) anthropogenic emissions in $g P_{2.5} \mathrm{~m}^{-2} \mathrm{yr}^{-1}$. Average annual PM2.5 emissions 1997 to 2014 are from to GFED4.1s, or ECLIPSE GAINS 4a for 2010, CLE scenario (anthropogenic). 


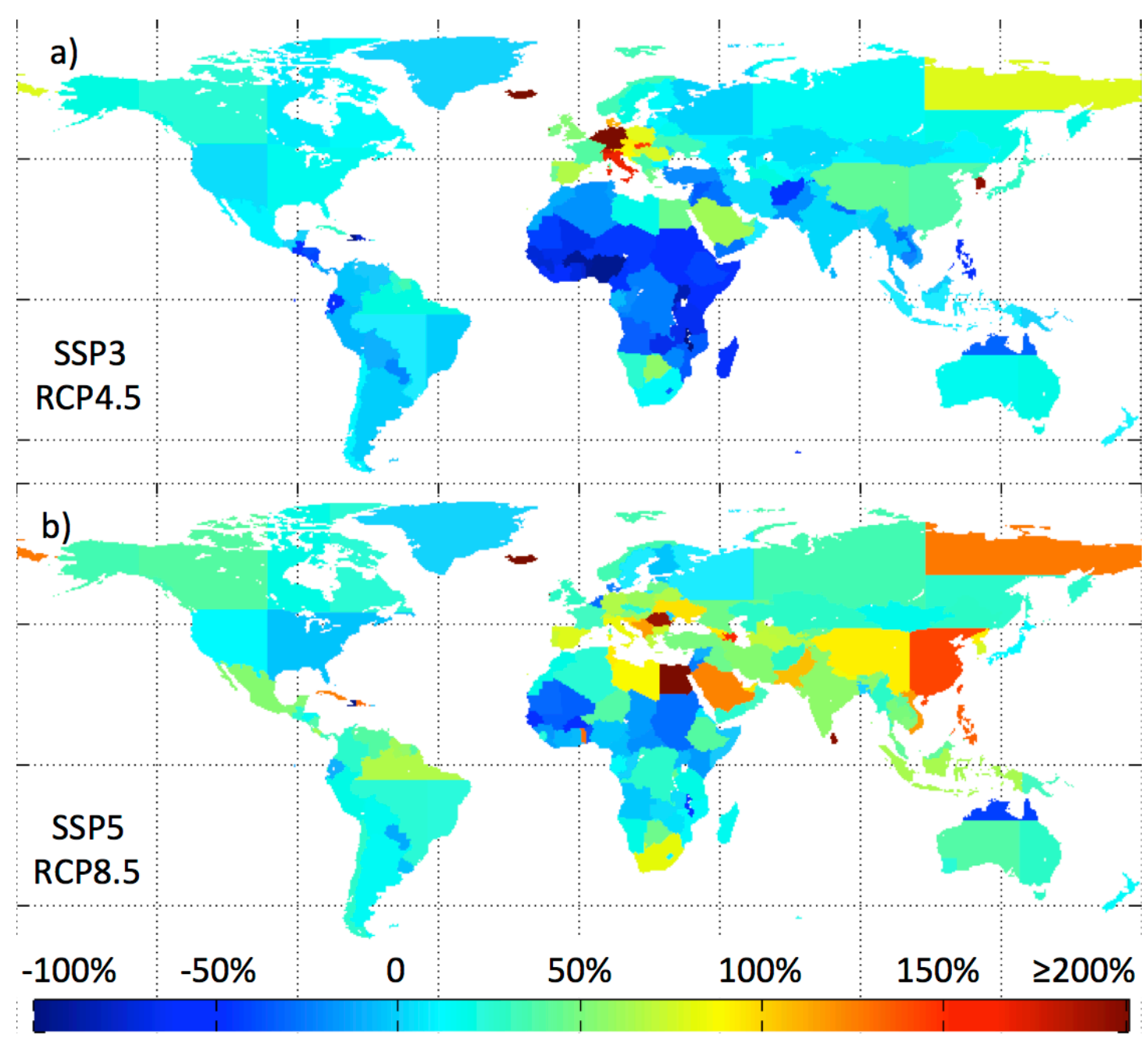

Figure S2: Relative change in annual PM2.5 emissions from current (1997-2014 mean) to 2090 (2080 to 2100 mean) by country/region. a) SSP3 globally high population growth (high-income countries: low population growth) with slow urbanisation and RCP4.5 climate scenario, b) SSP5 globally low population growth (high-income countries: high population growth) with slow urbanisation, RCP8.5 climate scenario. 


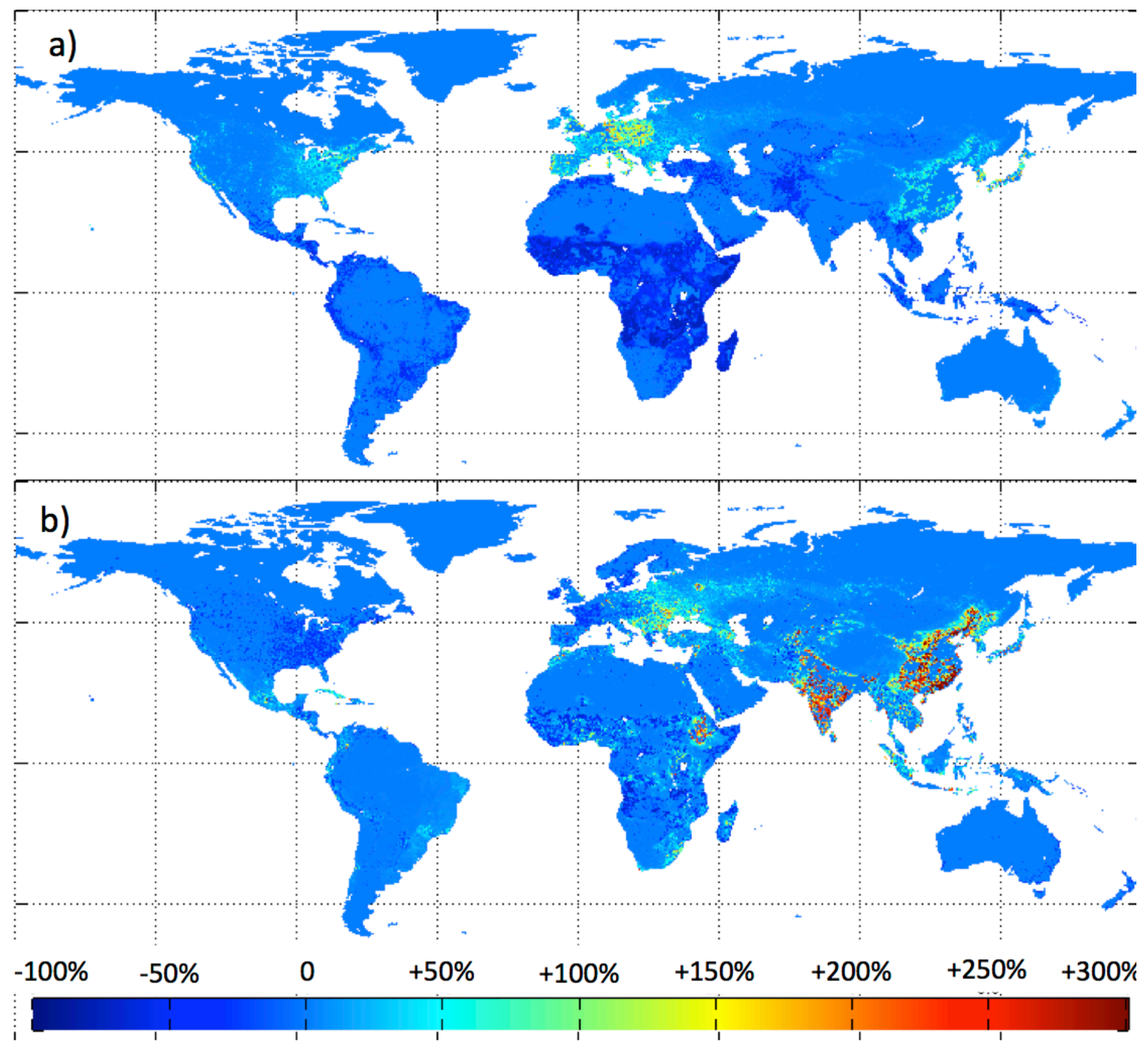

Figure S3: Relative change in wildfire emissions due to changes in population density only, between 2010 and 2090, according to Equ. 2. a) SSP3, b) SSP5 demographic scenario. 


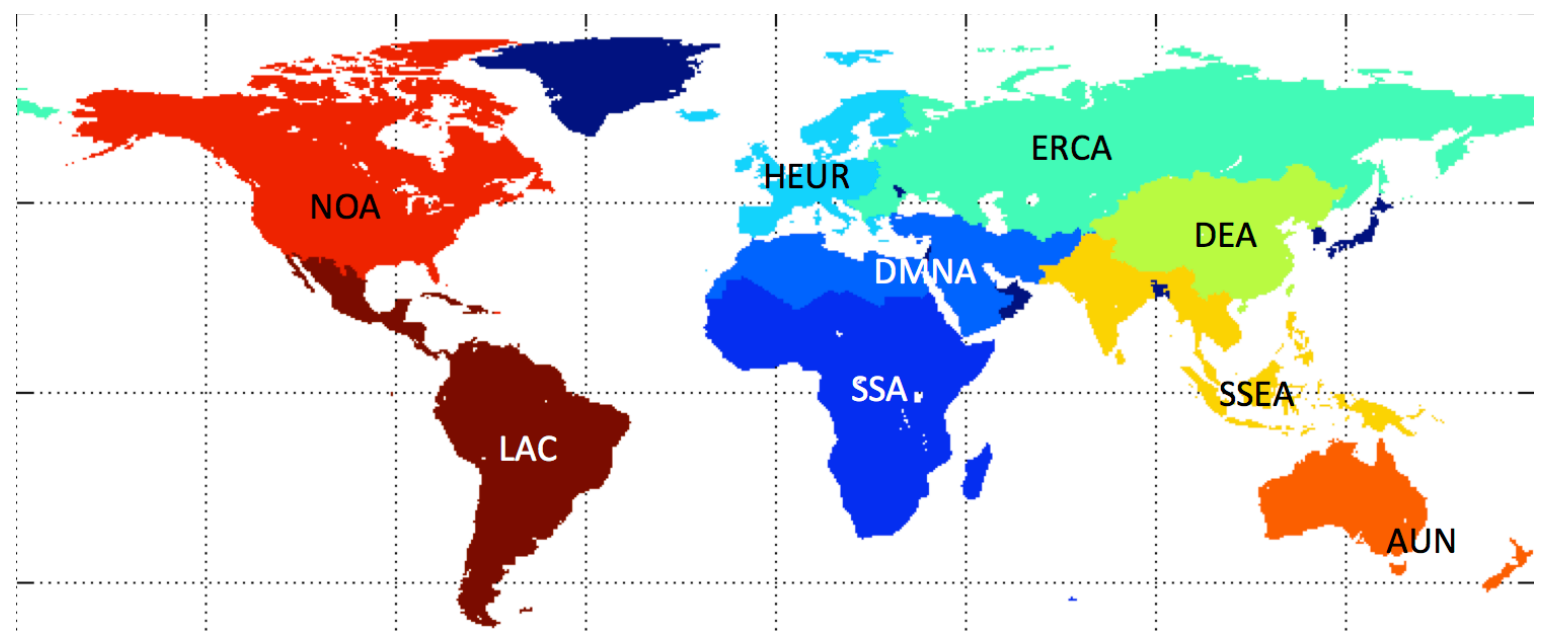

27 Figure S4: World regions used in the analysis. Dark blue: not included. 


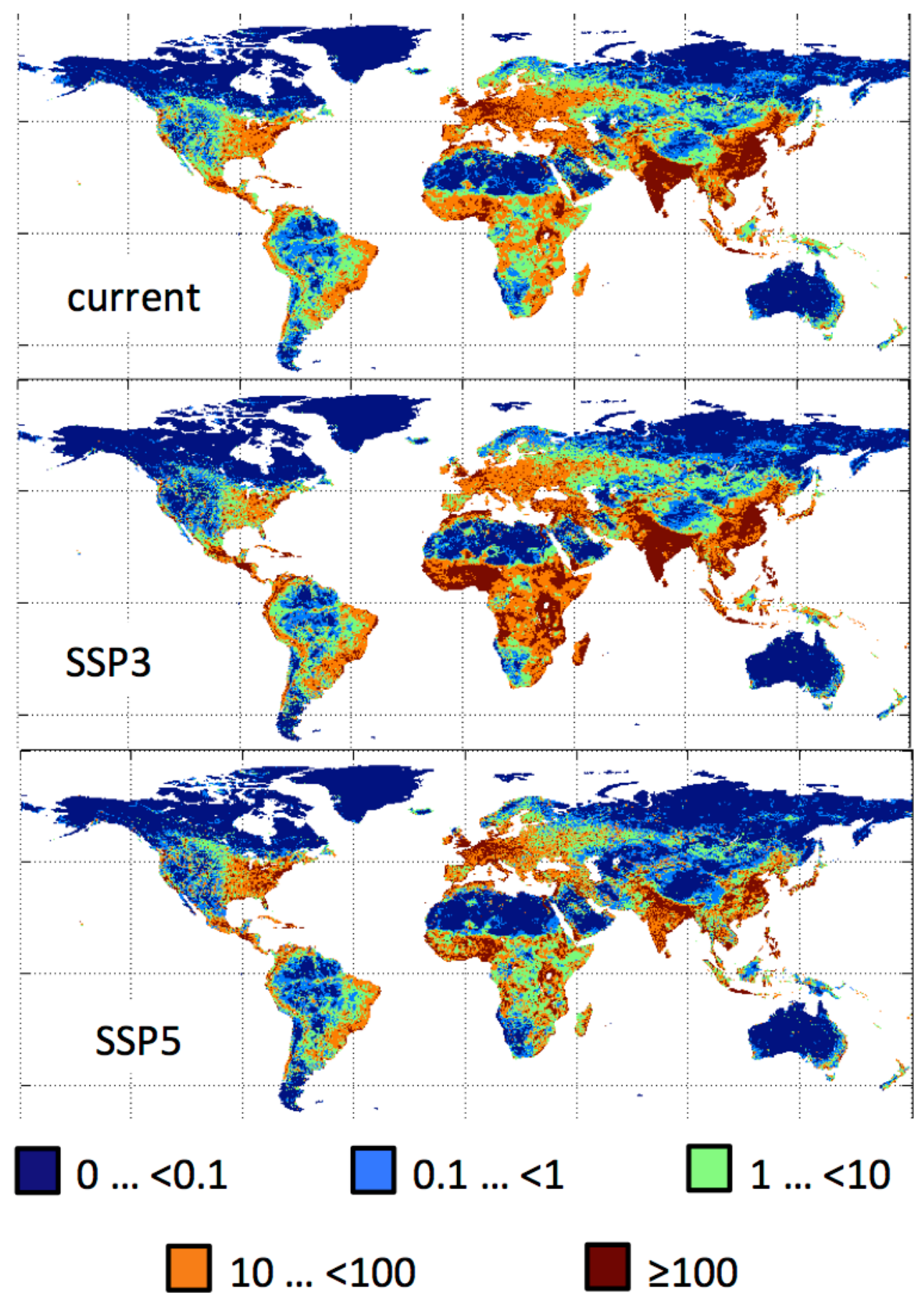
and SSP3 demographic scenarios. 